\title{
Phase Segregation in Cerium-Lanthanum Solid Solutions
}

\author{
V. Bellière, ${ }^{\dagger}$ G. Joorst, ${ }^{\dagger}$ O. Stephan, ${ }^{\ddagger}$ F. M. F. de Groot, ${ }^{\dagger}$ and B. M. Weckhuysen $*, \dagger$ \\ Inorganic Chemistry and Catalysis, Department of Chemistry, Utrecht University, Sorbonnelaan 16, \\ 3584 CA Utrecht, The Netherlands, and Laboratoire de Physique des Solides, Université Paris-Sud, \\ 91405 Orsay Cédex, France
}

Received: February 10, 2006; In Final Form: March 29, 2006

\begin{abstract}
Electron energy-loss spectroscopy (EELS) in combination with scanning transmission electron microscopy (STEM) reveals that the La enrichment at the surface of cerium-lanthanum solid solutions is an averaged effect and that segregation occurs in a mixed oxide phase. This separation occurs within a crystalline particle, where the dopant-rich phase is located at the surface of the dopant-deficient phase. The limiting structure appears to be a solid solution with a La fraction of $x=0.6$ in the bulk and $x=0.75$ at the surface. Up to a La fraction of 0.6, this phase will coexist with a lanthanum-type structure in different proportions depending on the dopant amount. STEM-EELS appears to be a powerful technique to clarify the existence of a multiphase system, and it shows that XRF, XPS, and XRD measure averaged results and do not show the phase complexity of the solids.
\end{abstract}

\section{Introduction}

Cerium-lanthanum-based compounds have become key components in the formulation of several commercial materials. They are widely used as catalysts for reducing $\mathrm{CO}$, hydrocarbons, and $\mathrm{NO}_{x}$ emissions from gasoline engines; ${ }^{1-4}$ for soot oxidation $;^{5}$ and as electrolyte materials of solid oxide fuel cells ${ }^{6}$ but also as sorbents for desulfurization processes. ${ }^{7}$ In the field of environmental catalysis, lanthanum oxide is well-known as surface stabilizer of supports based on alumina and zirconia, ${ }^{8,9}$ while the catalytic activities of pure and doped cerium have been associated with, for instance, interstitial oxides, ${ }^{10,11}$ lattice oxygen atoms, ${ }^{12,13}$ structural defects, ${ }^{14,15}$ basicity of surfaces, ${ }^{16}$ and redox activity. ${ }^{3,9,17,18}$ In most of these studies, catalytic activities have been reported as a function of the bulk composition of cerium-lanthanum mixed oxides, and only a few studies have focused on correlating catalytic activities to both bulk and surface composition. ${ }^{18,19}$ It appeared that the activity depends on the mixed oxide composition, which implies that the results will be different whether they are expressed as a function of the chemical composition of the bulk or of the surface.

Cerium crystallizes in the cubic fluorite structure, with cations in 8-fold and anions in 4-fold coordination. Lanthanum crystallizes in the A-type hexagonal structure and consists of layered $[\mathrm{LaO}]_{n}{ }^{n+}$ cations separated by discrete layers of $\mathrm{O}^{2-}$ anions to give $\mathrm{La}$ a 7 -fold coordination. The layers of $[\mathrm{LaO}]_{n}{ }^{n+}$ are formed from edge-sharing $[\mathrm{OLa}]_{4}$ tetrahedra disposed in a twodimensional hexagonal array perpendicular to the $c$-axis. Mixed cerium-lanthanum oxides, $\mathrm{Ce}_{1-x} \mathrm{La}_{x} \mathrm{O}_{2-x / 2}$, form solid solutions in either the fluorite or the A-type lanthanum oxide structure. However, these systems are much more complex than usually thought. Solubility limits for lanthanum oxide are usually quoted between 40 and $70 \% .^{20,21}$ Wilkes and co-workers have shown that, with cerium-lanthanum mixed oxides prepared by a precipitation method, over the major part of the composition

\footnotetext{
* Corresponding author. Phone: +31 30253 4328; fax: +31 30251 1027; e-mail: b.m.weckhuysen@chem.uu.nl.

$\dagger$ Utrecht University.

+ Université Paris-Sud.
}

range, up to $x=0.6$ lanthanum forms a solid solution in cerium; above $x=0.9$, cerium forms a solid solution in lanthanum; and the range between $x=0.6$ and $x=0.9$ results in the formation of mixed phases. ${ }^{22}$ Lanthanum and cerium exhibit preferential segregation to the surface of their solid solutions in cerium and lanthanum, respectively. This phenomenon has been attributed to changes in segregation energy with surface coverage because of solute-solute interactions. ${ }^{23}$ However, these conclusions result in a statistical-mechanical model, and no phase characterization in the intermediate range was possible.

Electron energy-loss spectroscopy (EELS) in combination with scanning transmission electron microscopy (STEM) offers a unique opportunity to correlate spectroscopic information with detailed structural knowledge of a material. ${ }^{24,25}$ In STEM-EELS, a quasi-monochromatic beam of electrons is incident on the sample, and the energy loss of the beam through the material is measured. The data provide detailed information about the chemical composition via the acquired core level EELS spectra, whereas the STEM images accurately map the material under study. STEM-EELS allows us to construct elemental and chemical (valence) maps with a spatial resolution of $0.5 \mathrm{~nm}$.

The goal of the present contribution is to evidence the surface segregation of lanthanum and cerium ions in cerium-lanthanum solid solutions and to identify the phases in the intermediate range. For this purpose, a series of cerium-lanthanum materials, $\mathrm{Ce}_{1-x} \mathrm{La}_{x} \mathrm{O}_{2-x / 2}$, has been prepared to the complete range of compositions and characterized by several techniques. Combining bulk and surface characterization techniques as well as techniques providing information on the long-range order and local order allows a detailed characterization of these promising solids. This has led to a better understanding of their structure and surface properties.

\section{Experimental Procedures}

Synthesis. A series of $\mathrm{Ce}_{1-x} \mathrm{La}_{x} \mathrm{O}_{2-x / 2}$ solid solutions, where $x=0,0.05,0.1,0.2,0.4,0.5,0.6,0.7,0.8,0.9$, and 1 , was prepared by solid-state reaction. Cerium(III) nitrate hexahydrate $\left(\mathrm{Ce}\left(\mathrm{NO}_{3}\right)_{3} \cdot 6 \mathrm{H}_{2} \mathrm{O}, 99.5 \%\right.$, Acros Organics) and lanthanum(III) 
nitrate hexahydrate $\left(\mathrm{La}\left(\mathrm{NO}_{3}\right)_{3} \cdot 6 \mathrm{H}_{2} \mathrm{O}, 98 \%+\right.$, Acros Organics) were mechanically mixed according to the desired composition and calcined under air at $1273 \mathrm{~K}$ for $2 \mathrm{~h}$ (heating rate of 10 $\mathrm{K} / \mathrm{min}$ ).

Characterization. X-ray fluorescence (XRF) data were obtained using a LAB 2000 from Spectro Analytical Instruments to determine the elemental bulk composition. Raman spectra were recorded with a Kaiser RXN Optical Spectrometer equipped with a holographic notch filter and transmission grating, a CCD camera, and $532 \mathrm{~nm}$ laser for excitation. All spectra were recorded with a data point resolution of $2 \mathrm{~cm}^{-1}$ and were measured in air at room temperature.

X-ray powder diffraction (XRD) data were collected for all samples at room temperature with a Bruker-Nonius PDS 120 powder diffractometer system, equipped with a position sensitive gas-filled detector of $120^{\circ} 2 \theta$, using cobalt $\mathrm{K}_{1}$ radiation $(\lambda=$ $1.78897 \AA$ ). Scans were measured over a $1 \mathrm{~h}$ period and compared with standard library patterns for phase analysis. The standards SRM1976 (alumina), SRM675 (mica), and SRM640 (silicon) were used for calibration. Lattice parameters were calculated and averaged over four peaks (i.e., (111), (200), (220), and (311)) using the cubic model.

The X-ray photoelectron spectroscopy (XPS) analysis was performed to determine the surface composition. The data were obtained with a Vacuum Generators XPS system, using a CLAM-2 hemispherical analyzer for electron detection. Nonmonochromatic $\mathrm{Al} \mathrm{K} \alpha \mathrm{X}$-ray radiation was used for exciting the photoelectron spectra using an anode current of $20 \mathrm{~mA}$ at $10 \mathrm{keV}$. The pass energy of the analyzer was set at $50 \mathrm{eV}$. The survey scan was taken with a pass energy of $100 \mathrm{eV}$. Lanthanum and cerium were quantified by measurement of the elementcharacteristic peak $\left(\mathrm{Ce}_{3 \mathrm{~d}}\right.$ and $\left.\mathrm{La}_{3 \mathrm{~d}}\right)$ areas following subtraction of a Shirley-type background. Relative sensitivities for the elements were calculated taking into account the photoelectron cross-section, angular asymmetry parameter, energy dependence of the inelastic mean free path, and transmission of the energy analyzer.

Scanning transmission electron microscopy (STEM) electron energy-loss spectroscopy (EELS) measurements were performed in Orsay (France) with a $100 \mathrm{keV}$ STEM instrument (VG HB 501) equipped with a field emission electron source. Mounted on the STEM instrument was a parallel electron energy-loss spectrometer (Gatan 666) optically coupled to a CCD camera generating an EELS spectrum with a $0.5-0.7 \mathrm{eV}$ energy resolution and $0.5 \mathrm{~nm}$ spatial resolution within a typical acquisition time down to $1 \mathrm{~ms}$ per pixel. ${ }^{24}$ The oxygen $\mathrm{K}$ edge as well as the cerium and lanthanum $\mathrm{M}_{4,5}$ (3d) edges were monitored. The solids were first sonicated in ethanol and then dropped on a holey amorphous carbon film supported on a copper grid. After the sample was scanned, appropriate areas were selected for the measurement of detailed 2-D STEM-EELS images. An energy range of $15 \mathrm{eV}$ has been used to quantify the EELS spectra (i.e., at the $\mathrm{La} \mathrm{M}_{5}$ edge $(832-847 \mathrm{eV})$ and at the $\mathrm{Ce} \mathrm{M}_{4}$ edge $\left.(902-917 \mathrm{eV})\right)$.

\section{Results and Discussion}

Figure 1 presents the Raman spectra of the whole ceriumlanthanum mixed oxide in the range of $360-520 \mathrm{~cm}^{-1}$. It can be noticed that cerium and lanthanum have both one characteristic Raman band. For cerium, the Raman band at $462 \mathrm{~cm}^{-1}$ is the only one allowed a lattice mode $\left(\mathrm{F}_{2 \mathrm{~g}}\right)$ of fluorite metal dioxides. ${ }^{26}$ This band is attributed to a symmetrical stretching vibration of the $\mathrm{Ce}-\mathrm{O} 8$ vibration unit, which is sensitive to any disorder in the oxygen sublattice. According to the literature,

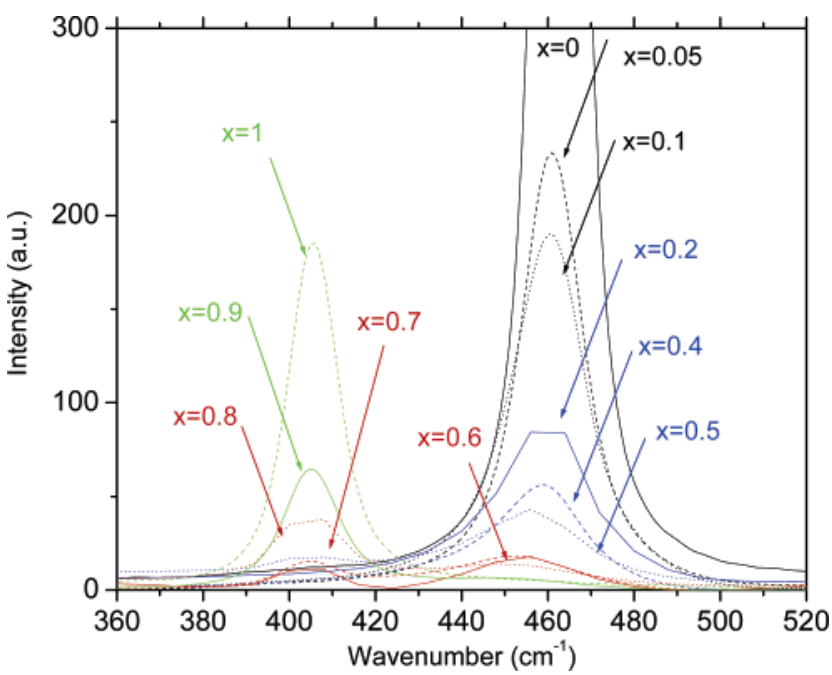

Figure 1. Raman spectra of $\mathrm{Ce}_{1-x} \mathrm{La}_{x} \mathrm{O}_{2-x / 2}$ solids. The spectrum with $x=0.0$ has a maximum of $\sim 850 \mathrm{au}$.

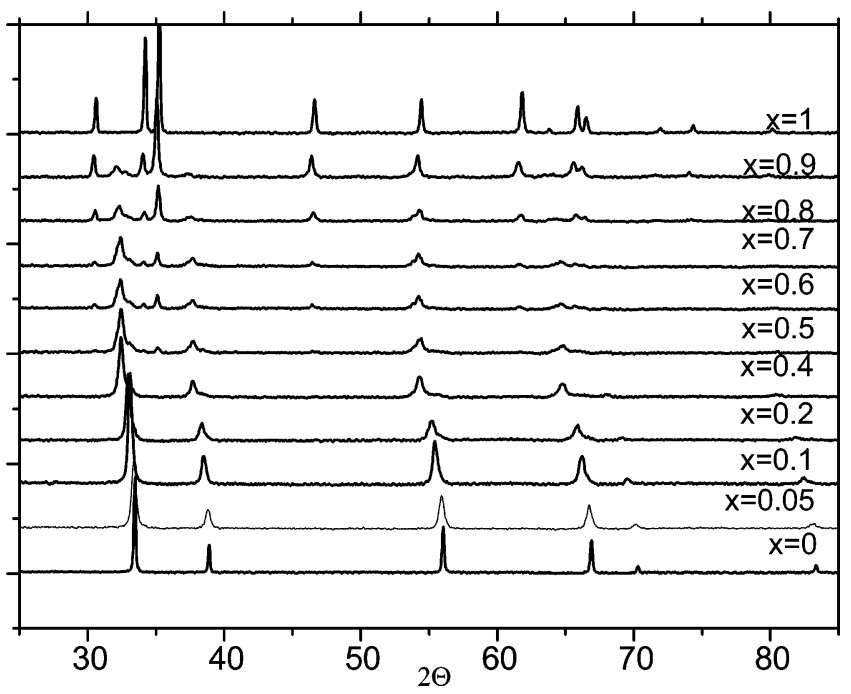

Figure 2. XRD patterns for $\mathrm{Ce}_{1-x} \mathrm{La}_{x} \mathrm{O}_{2-x / 2}$ solids where $0<x<1$.

A-type lanthanum Raman spectra have three characteristic bands at frequencies around $107 \mathrm{~cm}^{-1}\left(v_{4(\mathrm{Eg})}\right), 195 \mathrm{~cm}^{-1}\left(v_{2(\mathrm{~A} 1 \mathrm{~g})}\right)$, and $408 \mathrm{~cm}^{-1}\left(v_{3(\mathrm{Eg})}+v_{1(\mathrm{Alg})}\right) \cdot{ }^{27,28}$ The band at $107 \mathrm{~cm}^{-1}$ is often obscured by intense Rayleigh light. Thus, as shown in Figure 1, the pure lanthanum spectrum presents one Raman band at a frequency of $405 \mathrm{~cm}^{-1}$, which is attributed to a torsional skeleton mode of hexagonal units. Doping cerium with $\mathrm{La}^{3+}$ cations results in a decrease of the cerium Raman band intensity with a logical increase of the lanthanum Raman band. Moreover, the cerium Raman bands shift to lower frequencies (from 462 $\mathrm{cm}^{-1}(x=0)$ to $\left.450 \mathrm{~cm}^{-1}(x=0.8)\right)$ with the dopant rise. This lower frequencies shift of cerium bands indicates that cerium bonds become weaker with the insertion of $\mathrm{La}^{3+}$ into their lattice. Thus, the $\mathrm{La}^{3+}$ insertion in cerium results in the formation of cerium-lanthanum solid solutions and the deformation of its structure. This can be attributed to the difference in the cationic radii: $\mathrm{La}^{3+}, 103.2 \mathrm{pm}$ and $\mathrm{Ce}^{4+}, 87 \mathrm{pm}$.

Figure 2 shows the XRD patterns of the materials. On the pure cerium diffractogram, the peaks can be indexed to (111), (200), (220), (311), (222), (400), (331), (420), and (422) corresponding to a fluorite-structured cerium phase (space group $F m \overline{3} m$ ) with a lattice constant of $a=0.538 \mathrm{~nm}$ (JCPDS (Joint Committee on Powder Diffraction Standard) 75-0076). On the pure lanthanum diffractogram, the diffraction lines are attributed 


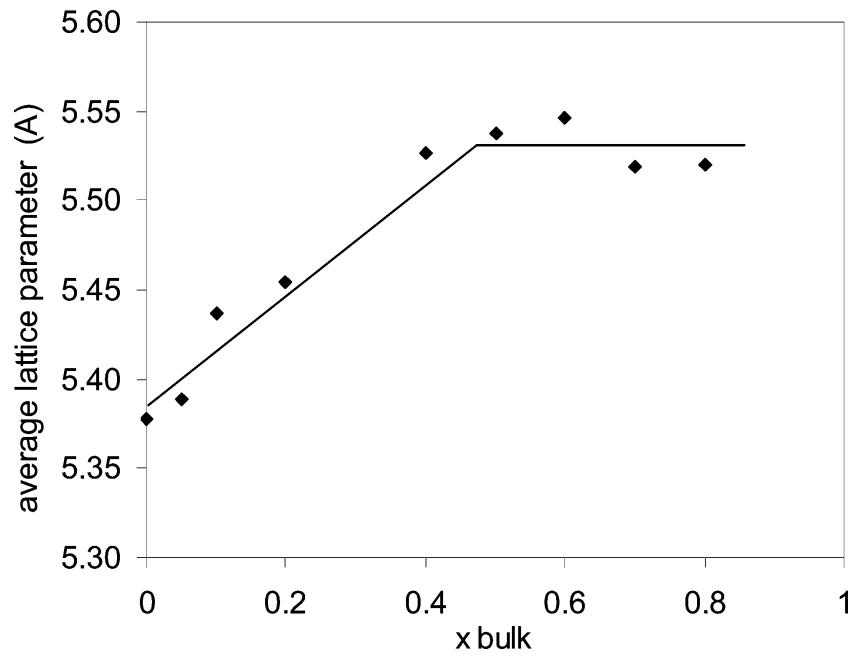

Figure 3. Influence of the bulk composition on the average lattice parameter of the cerium fluorite structure. The lines drawn are just guides for the eyes and do not correspond to any model.

to the hexagonal structure of lanthanum oxide (space group $P \overline{3} \mathrm{~m} 1$ ) with lattice constants of $a=0.3945 \mathrm{~nm}$ and $c=0.6151$ $\mathrm{nm}$ (JCPDS 02-0688). The first peaks can be indexed to (100), (002), (101), (102), (110), (103), (200), (201), (004), and (202). Therefore, cerium and lanthanum present face-centered cubic (fcc) and hexagonal close-packed (hcp) structures, respectively.

As illustrated in Figure 2, the pure fcc fluorite structure is retained up to an ionic fraction of about 0.5 in lanthanum, while the pure hcp structure of lanthanum is formed above an ionic fraction of 0.9. The intermediate compositions (i.e., between an ionic fraction of 0.5 and 0.9 in lanthanum) form mixed phases. These results are in good agreement with the work of Wilkes et al., who have shown theoretically that the pure oxides are only partially soluble in each other. ${ }^{23}$ The range limits appeared slightly different, probably because of the different preparation methods. Furthermore, the peak shift in the range between an ionic fraction of 0 and 0.5 in lanthanum suggests that the lanthanum insertion in cerium deforms its structure, confirming the Raman analysis. This is even more evident when the average lattice parameter of the cerium structure is plotted versus bulk composition (Figure 3). This average lattice parameter increases regularly up to an ionic fraction of about 0.5 in lanthanum. After this composition, it is difficult to determine, whereas the lattice parameter decreases or remains constant because of the broadening of the characteristic peaks used to estimate the lattice parameter. Nevertheless, according to the lattice parameter values, no pure cerium phase should exist up to an ionic fraction of about 0.5 in lanthanum, even in the presence of the lanthanum hexagonal structure.

Figure 4 presents the XPS results to determine the surface ionic fractions of cerium and lanthanum, where the probing depth of XPS at $\sim 700 \mathrm{eV}$ is $\sim 1.5 \mathrm{~nm}$. It is clear that below $x$ $=0.8$, the solid solutions are enriched in lanthanum at their surface. In summary, it seems that $x<0.5$ results in lanthanum dissolved in cerium with lanthanum segregation at its surface, $x>0.9$ results in cerium dissolved in lanthanum with some cerium segregation at its surface, and $0.5<x<0.9$ results in mixed phases (i.e., lanthanum dissolved in cerium with lanthanum segregation at its surface and cerium dissolved in lanthanum with cerium segregation at its surface).

To confirm these conclusions and to identify the intermediate phases, STEM-EELS measurements were performed on five solids: pure cerium, pure lanthanum, and mixed oxides with ionic fractions of $0.4,0.6$, and 0.9 in lanthanum. The pure solids

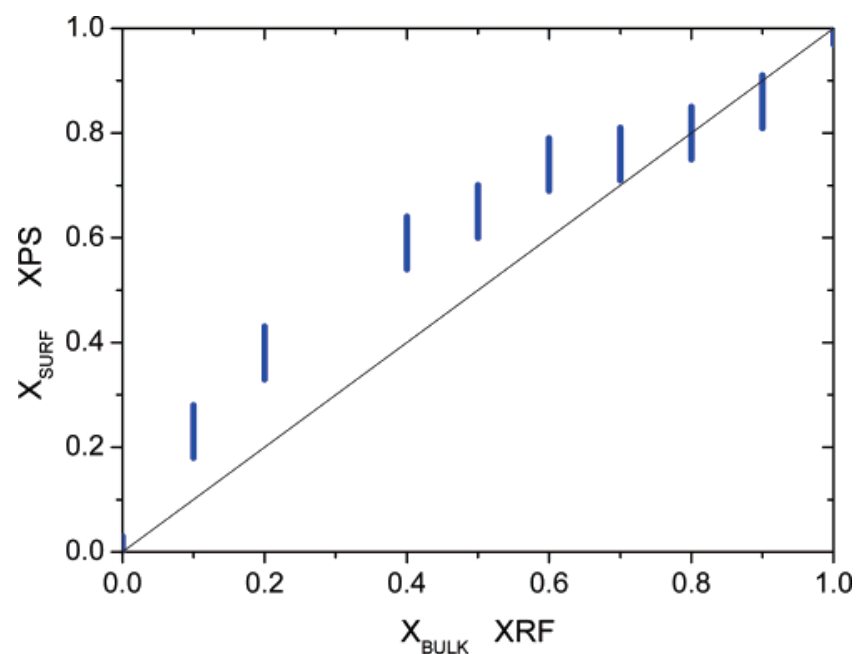

Figure 4. Composition of the surface as a function of the composition of the bulk mixed oxide in $\mathrm{Ce}_{1-x} \mathrm{La}_{x} \mathrm{O}_{2-x / 2}$ solids. The solid line represents the trend without any surface segregation. The uncertainty in the $X_{\mathrm{BULK}}$ value as determined from XRF is smaller than \pm 0.01 , and the uncertainty in the $X_{\mathrm{SURF}}$ value as determined from XPS is \pm 0.05 , as indicated by the symbols.

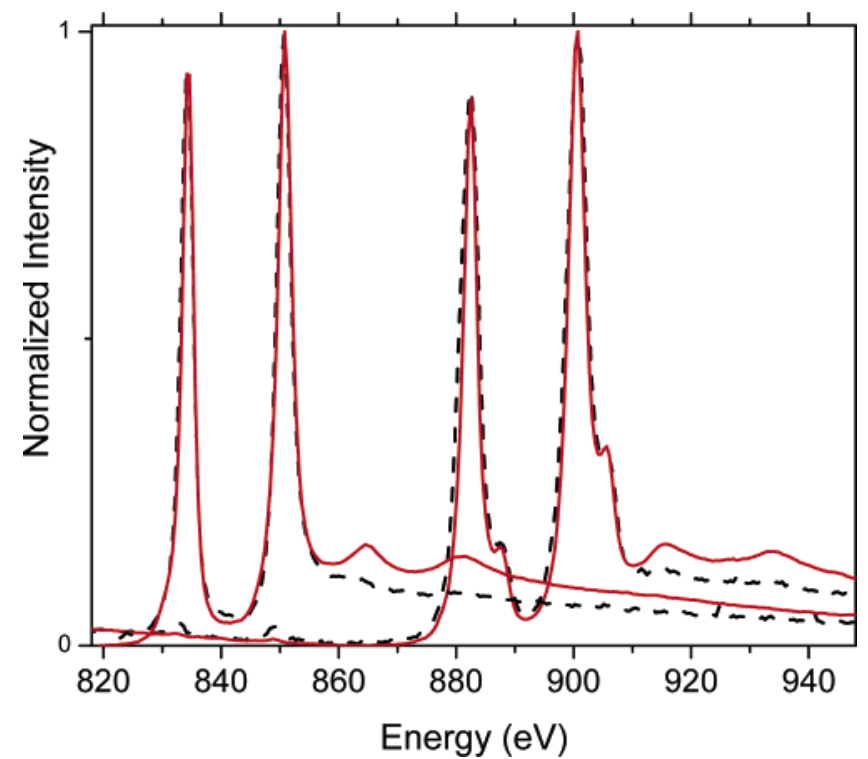

Figure 5. EELS spectra of pure $\mathrm{La}_{2} \mathrm{O}_{3}\left(\mathrm{La}^{3+}\right)$ and pure $\mathrm{CeO}_{2}\left(\mathrm{Ce}^{4+}\right)$. The $\mathrm{La}_{2} \mathrm{O}_{3}$ spectra have peaks at 832 and $850 \mathrm{eV}$. The $\mathrm{CeO}_{2}$ spectra have peaks at 883 and $902 \mathrm{eV}$. The (black) dashed line corresponds to extremely thin parts of the samples. The (red) solid line corresponds to a larger thickness in both samples.

were used as references, particularly for the oxidative state of the elements. Figure 5 shows the spectra of the lanthanum hexagonal structure $\left(\mathrm{La}^{3+}\right)$ in $\mathrm{La}_{2} \mathrm{O}_{3}$ and the cerium cubic structure $\left(\mathrm{Ce}^{4+}\right)$ in $\mathrm{CeO}_{2}$, respectively. The $\mathrm{Ce}$ and $\mathrm{La} \mathrm{M}_{4,5}$ edges are at 883 and $832 \mathrm{eV}$, respectively. Both cerium and lanthanum present four structures, where the two intense structures correspond to the $3 \mathrm{~d} \rightarrow 4 \mathrm{f}$ transitions of the $\mathrm{M}_{5}$ and $\mathrm{M}_{4}$ edges. The two small peaks that appear $\sim 30 \mathrm{eV}$ above the respective main peaks are due to electron multiple scattering (EMS) excitations of the core level plus a $\sim 30 \mathrm{eV}$ low-energy plasmon excitation. Quantitative analysis of the mixed oxides is hampered by the overlap of the $M_{4}$ EMS peak of lanthanum and the $M_{5}$ peak of cerium and by the associated difficulties of background correction for the cerium contributions. As a fair compromise, cerium and lanthanum have been quantified by measuring the $15 \mathrm{eV}$ energy range starting from the cerium $\mathrm{M}_{4}$ peak area at 

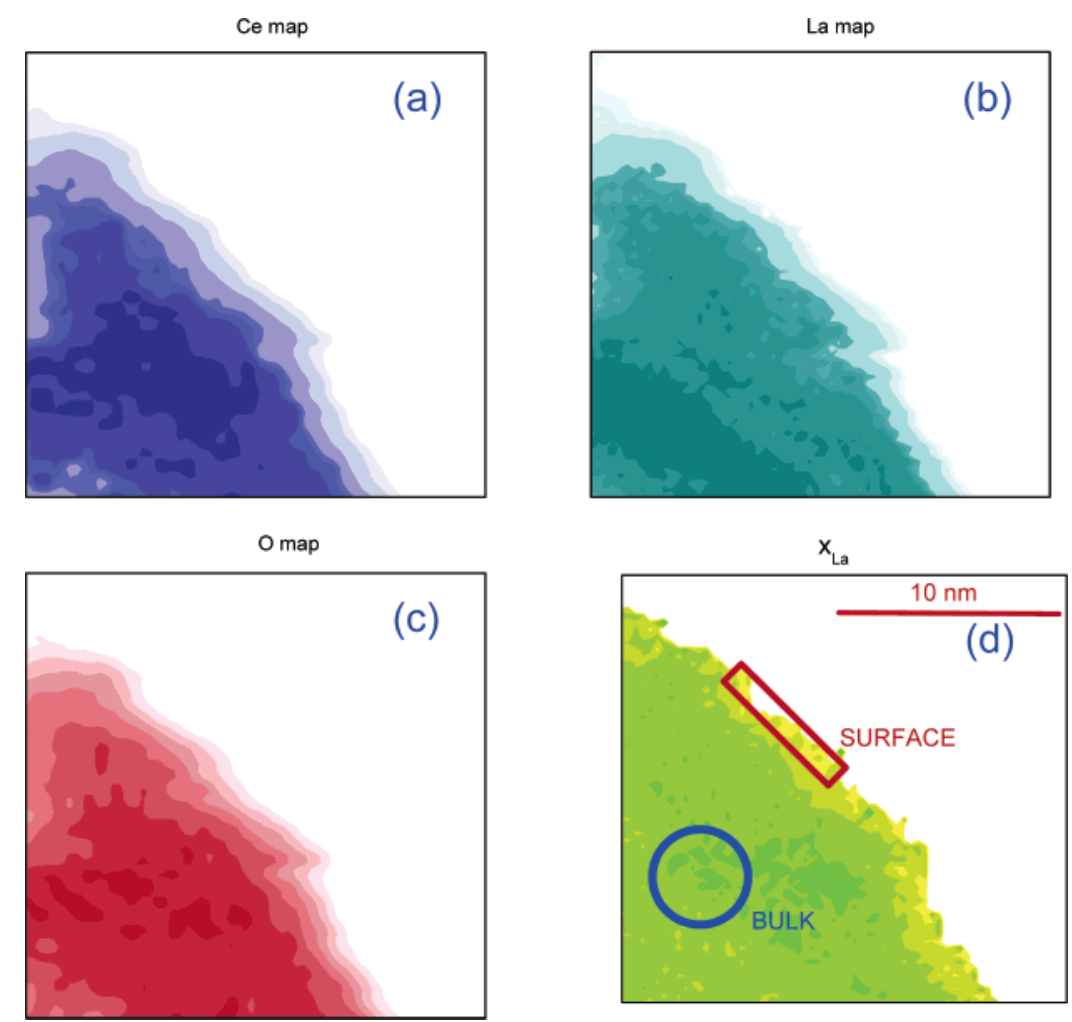

Figure 6. $20 \mathrm{~nm} \times 20 \mathrm{~nm}$ STEM-EELS images: (A) Ce map, (B) La map, (C) O map, and (D) $x_{\mathrm{La}}$ map ratio of $\mathrm{Ce}_{1-x} \mathrm{La}_{x} \mathrm{O}_{2-x / 2}$, with a bulk value of $x=0.38$. Color contours vary from values of 0-25000 (step of 5000) in panel A, from 0 to 15000 (step of 2500) in panel B, from 0 to 40000 (step of 5000) in panel C, and from $x_{\mathrm{La}} 0.3-0.7$ in panel D. The scale is identical in all images as indicated in panel D. Panel D indicates schematically the regions of the image that have been used to generate the EELS spectra as given in Figure 7.

$902 \mathrm{eV}$ (that has no contribution from lanthanum) and starting from the lanthanum $\mathrm{M}_{5}$ peak area at $832 \mathrm{eV}$. A background subtraction has been done for lanthanum contributions only. Relative sensitivities for the elements were calculated taking into account their cross-sections.

Chemical maps were generated from the EELS spectra to provide information on the chemical composition in the mixed oxides along the scanned areas. Figure 6 shows chemical maps obtained for the mixed oxide with a bulk ionic fraction of 0.4 in lanthanum. The darker the color in the chemical map, the larger the amount of the corresponding element. In Figure 6D, the blue color reflects the bulk cerium content of $0.35-0.45$, indicating that the bulk is homogeneous in composition. The green color reflects the surface enhancement of the fraction of La up to values between 0.6 and 0.7. The EELS spectra corresponding to these areas are given in Figure 7. These spectra are integrated over a certain bulk surface area, as has been schematically indicated in Figure 6D. The actual surface area is defined as the first $1.0 \mathrm{~nm}$ of the crystalline edge. The bulk spectrum is integrated over the entire bulk, defined as the whole area except the last $3.0 \mathrm{~nm}$ at the crystalline edge. It is noted that each pixel of the chemical maps is proportional to the number of atoms of the element of interest detected within the $0.5 \mathrm{~nm}$ diameter beam through the crystal. At the crystal edge, the electron beam enters at a grazing incidence, and the contribution of the surface atoms will be close to $100 \%$. When the probe enters the particle at a central position, the surface contribution is smaller than $5 \%$ as only the first and last 1.0 $\mathrm{nm}$ contributes to the surface and the remaining, approximately, $50.0 \mathrm{~nm}$ contributes to the bulk.

The EELS spectra in Figure 7 show a bulk ionic fraction of 0.38 in lanthanum and a surface ionic fraction of 0.6 in lanthanum, which is in line with the results of XRF and XPS analysis. The surface area is chosen to be $\sim 1 \mathrm{~nm}$, in line with

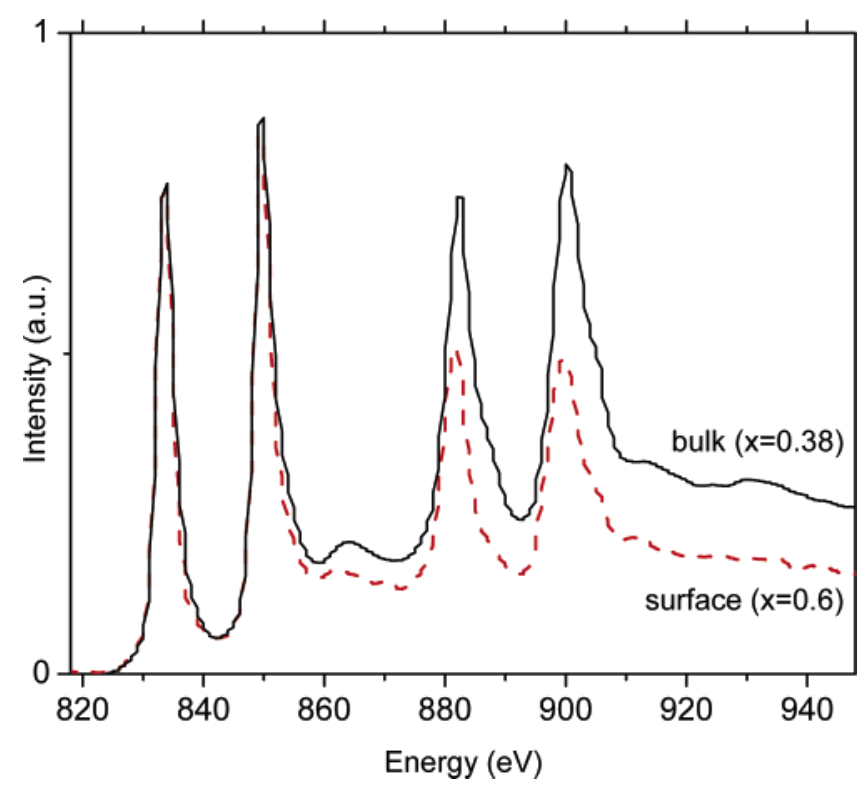

Figure 7. Integrated EELS spectra corresponding to the bulk (solid) and surface (dashed) areas of Figure 6; normalized at the $\mathrm{La} \mathrm{M}_{4,5}$ peaks.

the probing depth of XPS. The fine structure of the core-loss signals is identical to Figure 5, and it shows that the ions are, respectively, $\mathrm{La}^{3+}$ and $\mathrm{Ce}^{4+}$ as found in, respectively, $\mathrm{La}_{2} \mathrm{O}_{3}$ and $\mathrm{CeO}_{2}$. In conclusion, this material is a solid solution of lanthanum in a $\mathrm{CeO}_{2}$ structure with a bulk composition of $x=$ 0.38 and an integrated surface composition of $x=0.6$. Figure 6 shows that at some spots the surface composition can go up to $x=0.7$.

Figure 8 shows chemical maps obtained for the mixed oxide with a bulk ionic fraction of 0.6 in lanthanum. The scale is 100 $\times 100 \mathrm{~nm}, 5$ times larger than Figure 6 shows. Darker colors 
(a)

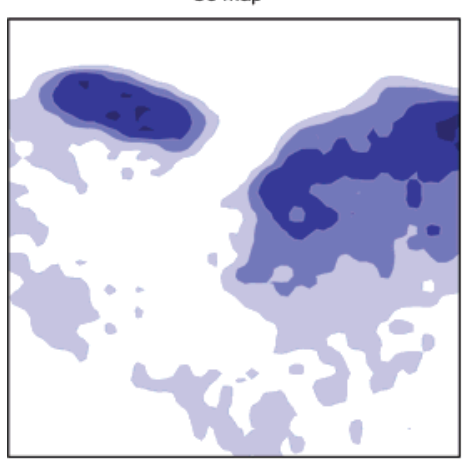

(c)

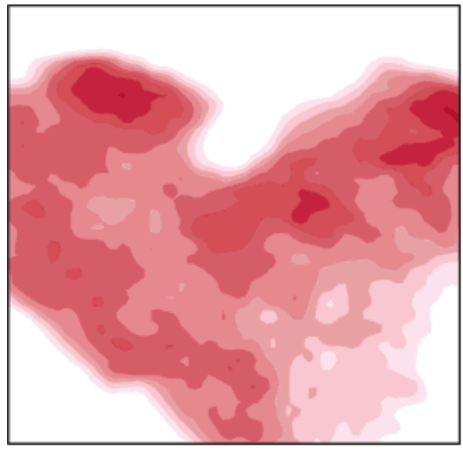

(b)

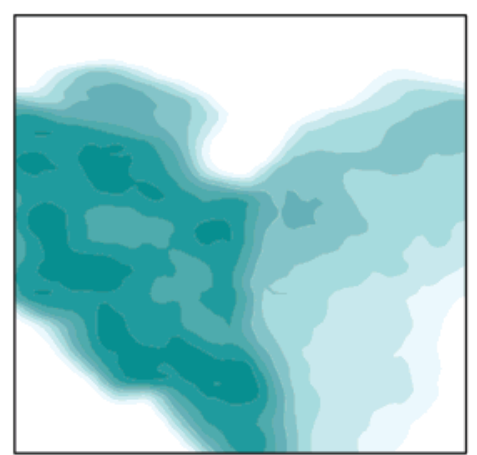

(d)

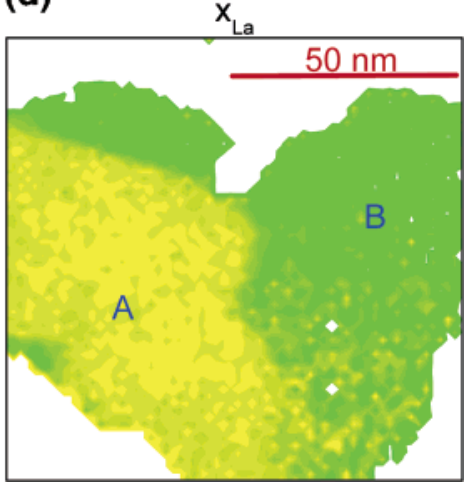

Figure 8. $100 \mathrm{~nm} \times 100 \mathrm{~nm} \mathrm{STEM-EELS}$ images showing (A) the Ce map, (B) the La map, (C) the O map, and (D) the $x_{\mathrm{La}}$ map of Ce $1-{ }_{x} \mathrm{La}{ }_{x} \mathrm{O}_{2-x / 2}$, with a bulk value of $x>0.9$ for area A and $x=0.6$ for area B in panel D. Color contours vary from values of $0-25000$ (step of 5000) in panel A, from 0 to 15000 (step of 2500) in panel B, from 0 to 40000 (step of 5000) in panel C, and from 0.5 to 0.9 (step of 0.05 ) in panel D.

reveal larger amounts of the corresponding element. Contrary to the prior solid, this mixed oxide contains two different phases: A and B. This is particularly visible in the $x_{\mathrm{La}}$ map, in which the yellow (light) region $\mathrm{A}$ has $x$ between 0.8 and 1.0, and the green (dark) region B has $x$ between 0.5 and 0.75 . The EELS spectra corresponding to these areas are given in Figure 9. Figure $9 \mathrm{~A}$ represents a particle containing mainly lanthanum with a mixed-oxide phase at its surface with an ionic fraction of 0.5 in lanthanum. Figure 9B represents a bulk ionic fraction of 0.6 in lanthanum and a surface ionic fraction of 0.75 in lanthanum. Because of the larger scale, these surface enhancements are difficult to see directly in Figure 8. This shows that this material presents two different phases: (i) a solid solution of lanthanum in $\mathrm{CeO}_{2}$ with a bulk composition of 0.6 in lanthanum and a surface composition of 0.75 in lanthanum and (ii) a solid solution of cerium in $\mathrm{La}_{2} \mathrm{O}_{3}$ with a bulk composition of 0.9 in lanthanum and a surface composition of 0.5 . These results show that the surface composition is always larger for the doped element.

In the case of the $x=0.9$ system, the maps (not shown) reveal that the mixed oxide contains again two different phases. The first phase is a particle of lanthanum oxide $(x=1.0)$. The second has a bulk ionic fraction of $x=0.6$ and a surface ionic fraction of $x=0.75$, corresponding to the phase B described previously for the mixed oxide with a bulk ionic fraction of $x=0.6$ (Figure $9 \mathrm{~B})$. We note that the exact numbers for the surface enhancement are directly related to the definition of the surface depth. In XPS, the escape depth of the electrons determines the surface depth. In our STEM-EELS analysis, we assign a certain thickness region to the surface. Figures 6 and 8 indicate that the surface value for $x$ reaches 0.75 only at the top $\sim 1 \mathrm{~nm}$ and also not over the complete surface. The presence of these two phases is corroborated by XRD measurements, although they are not detectable by the XRF and XPS analysis. The comparison of the XRD patterns of the mixed oxides (Figure 2) validates the coexistence of two kinds of phases: pure lanthanum oxide and solid solution with a bulk composition up to 0.6.

\section{Conclusion}

The multitechnique approach using Raman, XRF, XRD, XPS, and STEM-EELS presented in this work allowed us to identify the different phases of the $\mathrm{Ce}_{1-x} \mathrm{La}_{x} \mathrm{O}_{2-x / 2}$ system. To our knowledge, for the first time, STEM-EELS has been applied to lanthanum-cerium mixed oxides, and this study confirms the complexity of these systems.

The presence of a single fluorite $\left(\mathrm{CeO}_{2}\right)$ structure is maintained until the dopant ionic fraction reaches a value of about 0.5 in lanthanum. At this point, an A-type $\left(\mathrm{La}_{2} \mathrm{O}_{3}\right)$ hexagonal structure appears. The fluorite structure disappears up to an ionic fraction of 0.9 in lanthanum. The solid-state reaction at high temperature of the nitrate precursors leads to phase separation into dopant-rich and -deficient phases. STEM-EELS measurements reveal that the enrichment at the surface is not simply an enrichment of La as initially suggested by average results from $\mathrm{XRF}$ and XPS but a segregation into a mixed oxide phase. This separation occurs within a crystalline particle rather than separate particles, confirming the observation of extensive phase separation made by Morris and co-workers. ${ }^{21}$

All results are compared in Figure 10. XRD shows that there is only a $\mathrm{CeO}_{2} / \mathrm{La}$ system up to $x=0.5$ and only a $\mathrm{La}_{2} \mathrm{O}_{3} / \mathrm{Ce}$ system above $x=0.9$. Between, we assume a linear trend. The linear ratio of $\mathrm{CeO}_{2} / \mathrm{La}$ and $\mathrm{La}_{2} \mathrm{O}_{3} / \mathrm{Ce}$ also yields a linear trend for the surface composition as indicated by the dashed line. XPS shows an average surface enhancement against XRF, which is in line with the XRD predictions between $x=0.5$ and $x=0.9$. 

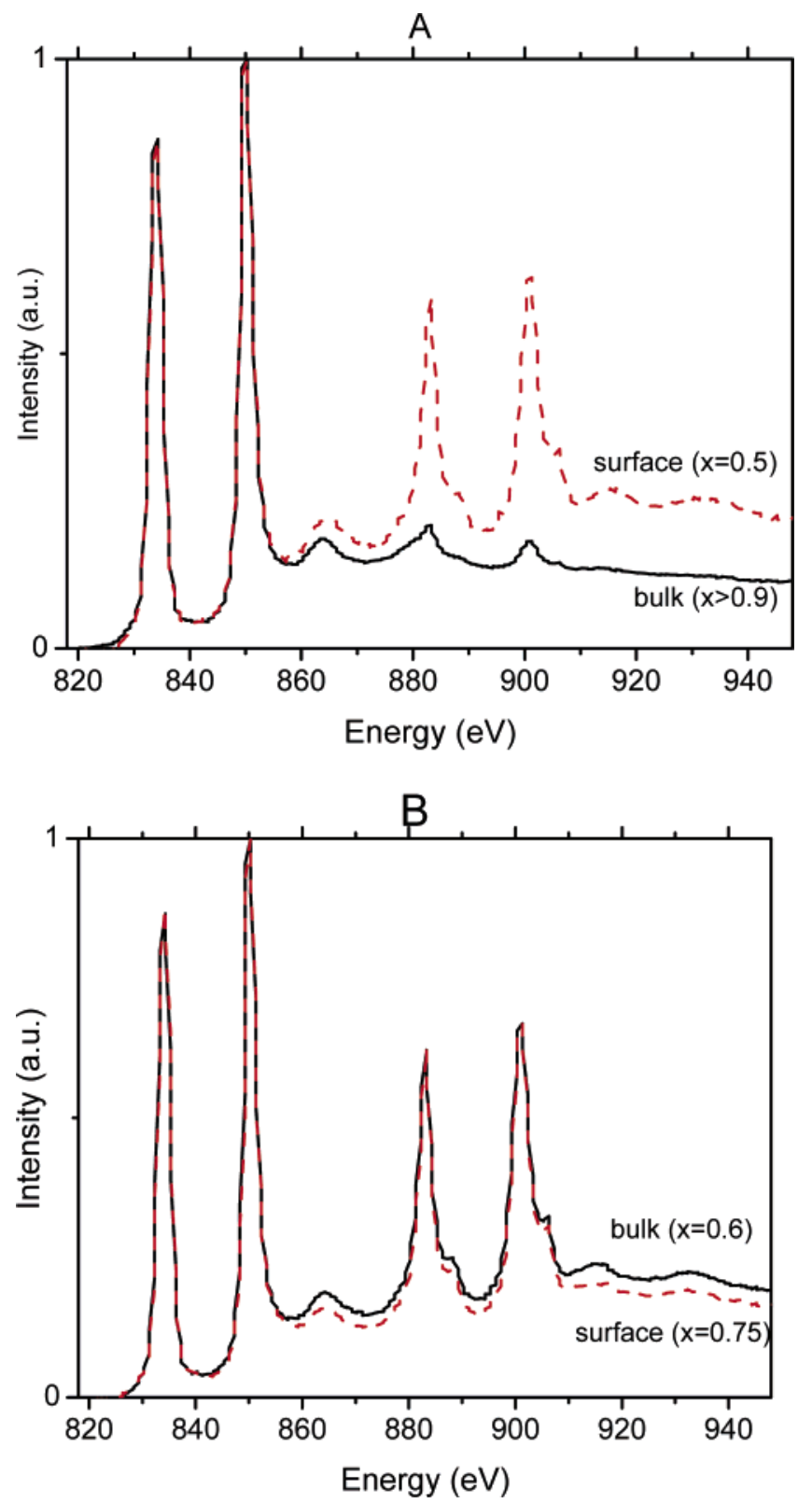

Figure 9. EELS spectra corresponding to the areas A and B presented in Figure $8 \mathrm{D}$ after normalization on the $\mathrm{La} \mathrm{M}_{4,5}$ bands.

STEM-EELS shows that the two phases show opposite surface enhancements. $\mathrm{CeO}_{2} / \mathrm{La}$ is enhanced in $\mathrm{La}$ at the surface, and $\mathrm{La}_{2} \mathrm{O}_{3} / \mathrm{Ce}$ is enhanced in Ce. The XPS result for $x=0.9$ confirms the surface enhancement of $\mathrm{Ce}$ in $\mathrm{La}_{2} \mathrm{O}_{3} / \mathrm{Ce}$. The encircled dashed area indicates that the surface (and bulk) fraction of $x$ is not a uniform value but shows some variation in $x_{\mathrm{BULK}}$ plus a large variation in $x_{\mathrm{SURF}}$, which also depends on the thickness assigned to the surface. In a mixed oxide, there will be two such regions, where the STEM-EELS results (cf. Figure 6) indicate that the saturated $\mathrm{CeO}_{2} / \mathrm{La}$ phase is rather uniform with $x_{\mathrm{BULK}}=0.6$ and $x_{\mathrm{SURF}}=0.75$. The EELS spectra show that in all systems, the valence of $\mathrm{Ce}$ is $4+$ (as in bulk $\mathrm{CeO}_{2}$, so with a fractional $4 \mathrm{f}$ count $^{29}$ ) and the valence of $\mathrm{La}$ is $3+$.

The lack of long-range order in cerium-lanthanum solid solutions has long been established. The data provided here clearly demonstrate that ordered phases are locally being formed. It is important to note that there is still not enough long-range order for these phases to be observed by means of XRD.

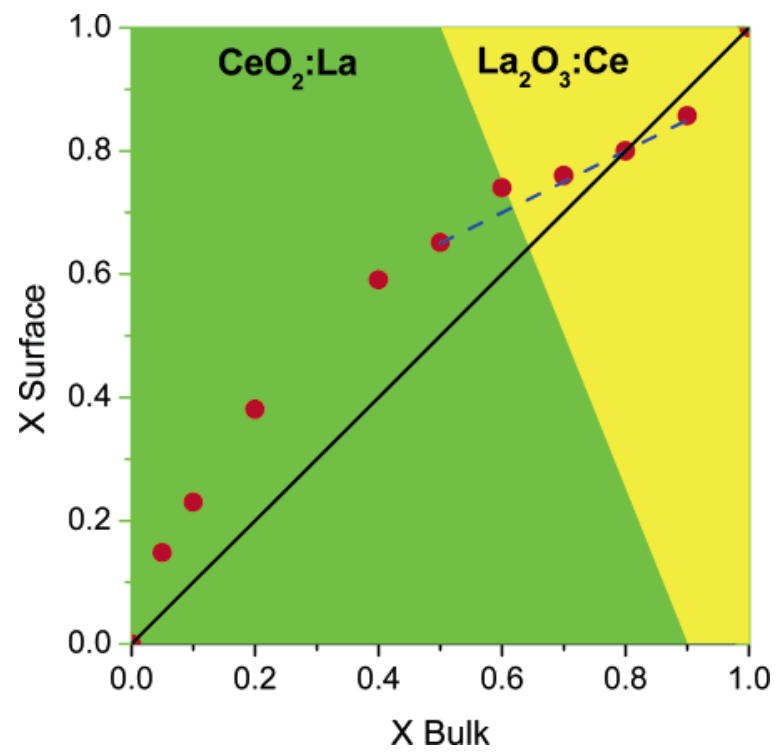

Figure 10. Combination of the results of XRD, XRF, XPS, and STEMEELS shows the comparison of the composition of the surface and the bulk for the mixed oxides in $\mathrm{Ce}_{1-x} \mathrm{La}_{x} \mathrm{O}_{2-x / 2}$ solids. The solid line gives the $x_{\mathrm{SURF}}=x_{\mathrm{BULK}}$ line. The green (dark) area is the $\mathrm{CeO}_{2} / \mathrm{La}$ system, and the yellow (light) area is the $\mathrm{La}_{2} \mathrm{O}_{3} / \mathrm{Ce}$ area. The dashed line is the interpolated $x_{\mathrm{SURF}}$. The red dots give the XPS results of $x_{\mathrm{SURF}}$ against the XRF results for $x_{\mathrm{BULK}}$. STEM-EELS shows surface enhancement of $\mathrm{La}$ in the $\mathrm{CeO}_{2} / \mathrm{La}$ system and $\mathrm{Ce}$ enhancement in the $\mathrm{La}_{2} \mathrm{O}_{3} / \mathrm{Ce}$ system.

To conclude, we have shown in this paper that these materials can be described as a dopant-deficient phase supporting dopantrich phases. Therefore, since catalysis is mainly a surface phenomenon, the dopant-rich phase must be the active phase in catalytic reactions. Thus, it is important to remember that caution must be taken with interpretations of the ceriumlanthanum solid solution characterizations. XRF, XPS, and XRD measure averaged results and do not show the phase complexity of the solids. STEM-EELS appears to be a powerful technique to clarify the existence of a multiphase system.

Acknowledgment. The authors gratefully acknowledge financial support from CW-NWO-VICI and the University of Paris-Sud for TEM-EELS measurements.

\section{References and Notes}

(1) Trovarelli, A.; de Leitenburg, C.; Boaro, M.; Dolcetti, G. Catal. Today 1999, 50, 353. 173.

(2) Bernal, S.; Kaspar, A.; Trovarelli, A. Catal. Today 1999, 50,

(3) O'Connell, M.; Morris, M. A. Catal. Today 2000, 59, 387.

(4) Trovarelli, A., Ed. Catalysis by Ceria and Related Materials; Imperial College Press: London, 2002.

(5) Bueno-Lopez, A.; Krishna, K.; Makkee, M.; Moulijn, J. A. J. Catal. $\mathbf{2 0 0 5}, 230,252$.

(6) Sahibzada, M.; Steele, B. C. H.; Zheng, K.; Rudkin, R. A.; Metcalfe, I. S. Catal. Today 1997, 38, 459.

(7) Wang, Z.; Flytzani-Stephanopoulos, M. Energy Fuels 2005, 19, 2089.

(8) Beguin, B.; Garbowski, E.; Primet, M. Appl. Catal. 1991, 75, 119

(9) Colussi, S.; de Leitenburg, C.; Dolcetti, G.; Trovarelli, A. J. Alloys Compd. 2004, 374, 387.

(10) Campbell, K. D.; Zhang, M.; Lunsford, J. H. J. Phys. Chem. 1988, 92,750 .

(11) Osada, Y.; Koike, S.; Fukushima, T.; Ogasawara, T.; Shikada, T.; Ikariya, T. Appl. Catal. 1990, 59, 59.

(12) Trovarelli, A. Catal. Rev. 1996, 38, 439.

(13) Ozawa, M.; Loong, C.-K. Catal. Today 1999, 50, 329.

(14) Zamar, F.; Trovarelli, A.; de Leitenburg, C.; Dolcetti, G. J. Chem. Soc., Chem. Commun. 1995, 965.

(15) O'Neill, W. M.; Morris, M. A. Chem. Phys. Lett. 1999, 305, 389. 
(16) Choudhary, V. R.; Rane, V. H. J. Catal. 1991, 130, 411.

(17) Hattori, T.; Inoko, J.-I.; Murukami, Y. J. Catal. 1976, 42, 60.

(18) Wilkes, M. F.; Hayden, P.; Bhattacharya, A. K. J. Catal. 2003 $219,286$.

(19) Wilkes, M. F.; Hayden, P.; Bhattacharya, A. K. J. Catal. 2003, $219,295$.

(20) Mogensen, M.; Sammes, N. M.; Tompsett, G. A. Solid State Ionics 2000, 129, 63

(21) Ryan, K. M.; McGrath, J. P.; Farrell, R. A.; O’Neill, W. M.; Barnes, C. J.; Morris, M. A. J. Phys.: Condens. Matter 2003, 15, L49.

(22) Wilkes, M. F.; Hayden, P.; Bhattacharya, A. K. J. Catal. 2003 219,305 .

(23) Wilkes, M. F.; Hayden, P.; Bhattacharya, A. K. Appl. Surf. Sci. 2003, 206, 12
(24) Stephan, O.; Gloter, A.; Imhoff, D.; Kociak, M.; Mory, C.; Suenaga, K.; Tence, M.; Colliex, C. Surf. Rev. Lett. 2000, 7, 475.

(25) Wang, Z. L. Adv. Mater. 2003, 15, 1497.

(26) McBride, J. R.; Hass, K. C.; Poindexter, B. D.; Weber, W. H. J. Appl. Phys. 1994, 76, 2435.

(27) Boldish, S. I.; White, W. B. Spectrochim. Acta, Part A 1979, 35 1235

(28) Weckhuysen, B. M.; Rosynek, M. P.; Lunsford, J. H. Phys. Chem. Chem. Phys. 1999, 1, 3157.

(29) Butorin, S. M.; Mancini, D. C.; Guo, J.-H.; Wassdahl, N.; Nordgren, J.; Nakazawa, M.; Tanaka, S.; Uozumi, T.; Kotani, A.; Ma, Y.; Myano, K. E.; Karlin, B. A.; Shuh, D. K. Phys. Rev. Lett. 1996, 77, 574. 